\title{
The Effect of Shared Information on Pilot/Controller and Controller/Controller Interactions
}

\author{
R. John Hansman and Hayley J. Davison \\ Department of Aeronautics \& Astronautics \\ MIT International Center for Air Transportation \\ Massachusetts Institute of Technology \\ Cambridge, MA 02139 USA \\ Tel: 617-253-2271, Fax: 617-253-4196,E-mail: rjhans@mit.edu
}

\begin{abstract}
The increased ability to exchange information between Pilots, Controllers, Dispatchers, and other agents is a key component of advanced Air Traffic Management. The importance of shared information as well as current and evolving practices in information sharing are presented for a variety of interactions including: Controller/Pilot interactions, Pilot/Airline interactions, Controller/Controller interactions, and Airline/ATM interactions.
\end{abstract}

\section{Introduction}

In order to respond to the increasing demand on limited airspace system resources, a number of applications of information technology have been proposed, or are under investigation, to improve the efficiency, capacity and reliability of $A T M$ operations. Much of the attention in advanced ATM technology has focused on advanced automation systems or decision aiding systems to improve the performance of individual Pilots or Controllers. However, the most significant overall potential for information technology appears to be in increasing the shared information between human agents such as Pilots, Controllers or between interacting Controllers or traffic flow managers. Examples of proposed shared information systems in the US include; Controller Pilot Datalink Communication (CPDLC), Traffic Management Advisor (TMA); Automatic Dependant Surveillance (ADS); Collaborative Decision Making (CDM) and NAS Level Common Information Exchange. [1]

Air Traffic Management is fundamentally a human-centered process consisting of the negotiation, execution and monitoring of contracts between human agents for the allocation of limited airspace, runway and airport surface resources. The decision processes within ATM tend to be Semistructured. Many of the routine elements in ATM decision making on the part of the Controllers or Pilots are well Structured and can be represented by well defined rules or procedures. However in disrupted conditions, the ATM decision processes are often Unstructured and cannot be reduced to a set of discrete rules. As a consequence, the ability to automate ATM processes will be limited and ATM will continue to be a human-centric process where the responsibility and the authority for the negotiation will continue to rest with human Controllers and Pilots. As the ATM system leans more towards distributed authority to improve efficiency and safety, the use of information technology to support the human decision process will therefore become an important aspect.

The premise of many of the proposed shared information systems is that the performance of ATM operations will improve with an increase in Shared Situation Awareness between agents (Pilots, Controller, Dispatchers). This will allow better informed control decisions and an improved ability to negotiate between agents. A common information basis may reduce communication load and increase the level of collaboration in the decision process.

In general, information sharing is expected to have advantages for all agents within the system. However there are important questions which remain to be addressed. För example: What shared information is most important for developing effective Shared Situation Awareness? Are there issues of information saturation? Does information parily create ambiguity in control authority? Will information sharing induce undesirable or unstable gaming behavior between agents?

This paper will explore the effect of current and proposed information sharing between different ATM agents. The paper will primarily concentrate on bilateral tactical interactions between specific agents (PilovController; Controller/Controller; PilotDispatcher: Controller/Dispatcher) however it will also briefly discuss multilateral interaction and more strategic interactions. The information about the interactions was based on field observations in air traffic control facilities, flight decks, and dispatch units as well as a series of surveys 
inquiring about the status of information sharing in the NAS today.

\section{Why Humans are Necessary in ATM}

The need for human decision makers within ATM can be demonstrated from an analysis of ATM decision processes based on recent decision theory models. [2] Semistructured decision theory asserts that humans add value to Unstructured decision processes. A Semistructured decision process consists of Structured and Unstructured subprocesses. A Structured decision process is defined as a process which can be reduced to a well defined set of rules and therefore could be reliably automated. Unstructured processes are those for which a reliable set of rules governing the decision process cannot be defined a priori. There are many reasons why it may be inappropriate to Structure a decision process including: insufficient understanding of the process due to complexity, ambiguity of goals, insufficient data on which to base a structured decision, uncertainty, and changing environments, as well as humanistic elements such as creativity or subjective and moral judgement.

Within ATM examples of Structured decisions are those completely defined by Standard Operating Procedures, routine processes or currently automated processes such as radar tracking algorithms. However, many important decisions within ATM are Unstructured and therefore difficult to reliably automate. Even relatively simple decision problems such as conflict resolution are difficult to automate using classical optimization techniques. More complex problems such as dealing with multi aircraft conflicts, irregular operations, and ambiguous situations tend to be even less structured and clearly require Unstructured solution strategies. It should be noted that decision processes are more likely to become Unstructured as the state of the system moves away from nominal operations. Therefore information systems which support the human in Unstructured decisions are most important in nonnominal situations due to irtegular operations, weather, unanticipated failures or emergency situations.

In Unstructured decision theory, it is not possible to define the optimal decision a priori but it is sometimes possible to identify well-informed or illinformed decision situations. In many cases, situations are unstructured simply because they lack sufficient data to execute a well-structured decision rule. Often, in these situations, the human is called upon to diagnose the situation and to infer the missing data or to project a probable future state of the system.

In general, the more informed the human agent, the better the decision process. However, if 100 much information is presented in time critical situations, the human can become saturated and may not be able to integrate important information due to distraction, fixation, or simple overload. Expert operators (Pilots and Controllers) demonstrate the ability to filter out non-critical information and have a higher tolerance to information saturation, however even this filtering process appears to be Unstructured.

In processes involving multiple humans, the ability to share information generally makes each agent more informed and can result in improved overall negotiations and decisions, particularly if the shared information supports a well-structured decision process with a clearly optimal course of action. One aspect which complicates multi-agent decision systems is resolving goal differences between the agents. In most Unstructured decisions, humans exhibit extremely complex goal sets. While agents may share a partial set of goals, there are elements in their goal sets which diverge. For example in a comparison of Controller and Pilot goal sets, Farley et al. $[3,4,5]$ found that Controllers and Pilots share the goal of maintaining safety of flight where they have differing goals with regard to optimizing the performance of the system. Controllers tend to have the goal of maintaining the flow within the sector while the Pilots have the objective to optimize the performance of their specific flight.

In cases of weakly differing goals, the decision/negotiation process may become difficult due to ambiguity where it may not be clear which goal set has the higher priority. In timeconstrained decisions such as tactical ATM it is therefore necessary to have a clearly defined hierarchy to resolve ambiguity. In more strongly differing goal sets, such as direct competition for resources, agents may exhibit gaming behavior where their actions may be strongly coupled to other agents in the system. Potential issues of undesirable gaming behavior must be considered.

Based on the semistructured nature of decision processes within ATM, as well as issues of responsibility and societal trust, it is clear that human Pilots, Controllers and Dispatchers will continue to have supervisory decision making responsibility in future ATM systems. $[6,7]$ In developing decision support and information systems, considerations on how the proposed 


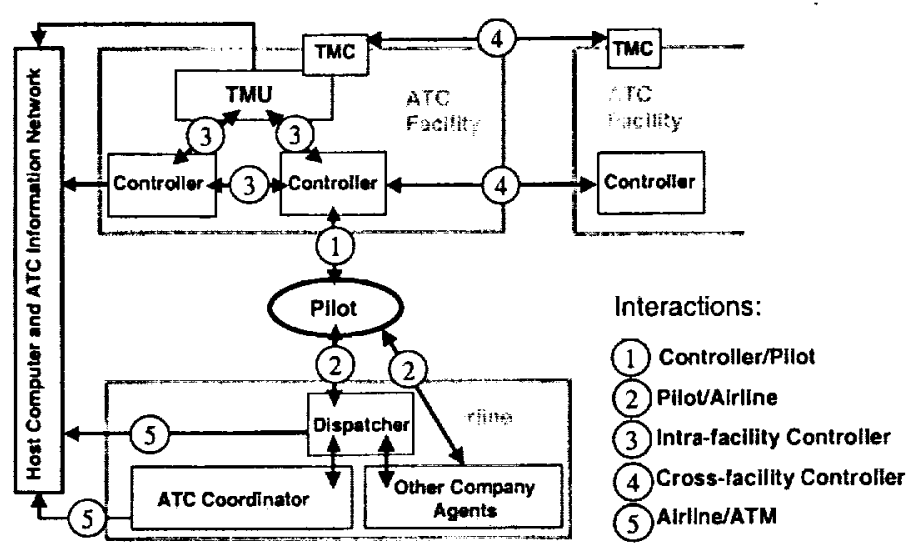

Figure 1: ATM Tactical Interaction Architecture.

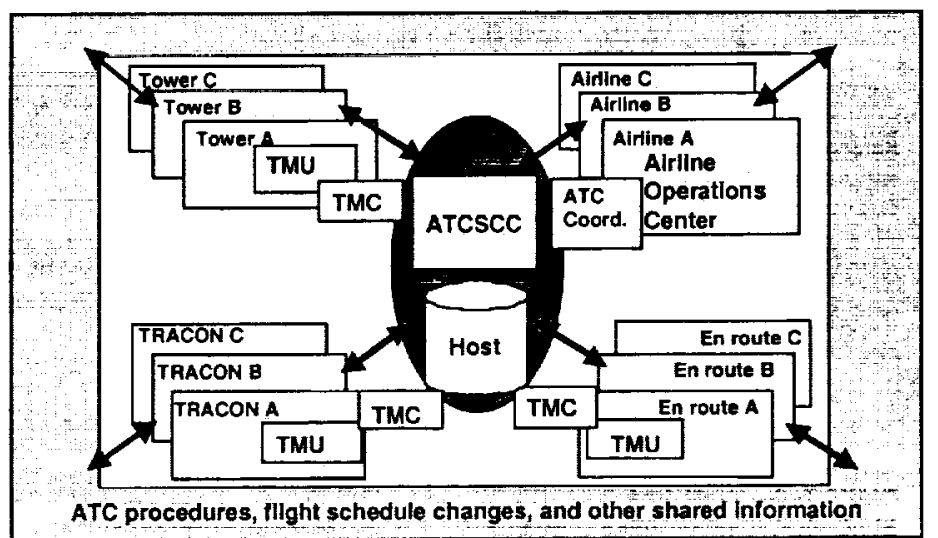

Figure 2: ATM Strategic Interaction Architecture.

systems will impact the interaction needs to be considered.

\section{ATM Interaction Architecture}

In order to discuss the impact of shared information it is useful to define the interaction architecture. The interactions between agents within ATM can be generally separated into tactical and strategic. The tactical level is focused on the management of individual flights as they propagate through the airspace systems. The key tactical interactions for a particular flight are shown schematically in Figure 1. The central interaction is between the Pilot and the current tactical Controller who is managing that flight, and this is shown as Interaction 1 in Figure 1. However the Pilot (for Airline operations) also interacts with other company representatives including the Dispatcher (Interaction 2) who provides technical and decision support services (e.g., flight planning, weights, weather) and other company agents (e.g., Station, Ramp, etc.) who interact with the flight. The tactical Controller also interacts with other Controllers who have current or future interest in the flight. In terms of the information architecture, it is important to distinguish which interactions occur within the same facility (Inter-Facility, Interaction 3) from those interactions which occur between facilities (Cross-Facility, Interaction 4). Within each facility there is a Traffic Management Coordinator (TMC) as a part of the Traffic Management Unit (TMU) who interacts with the tactical Controllers and is the principal external contact point for both tactical and strategic interactions with the facility. Performing a similar function for the airlines, the ATC Coordinator is responsible for the tactical and strategic interactions with ATM (Interaction 5) such as receiving information about NAS delays and conveying the delays to the Dispatchers as well as submitting and making changes to canned flight plans.

At the strategic level, the interactions, shown schematically in Figure 2, focus principally on the 
management of traffic flows and airspace system resources. In the US, the central elements of strategic interaction are performed by the Air Traffic Control System Control Center (ATCSCC). These elements interact with each of the control facilities (Centers, specified TRACONs and Towers) as well as with the airlines generically represented as Airline Operations Centers (AOC).

\section{Interaction Assumptions}

The interactions between human agents within the ATM system are based on an assumed set of common rules, information, procedures, background, language and culture. The common rules are the operating regulations such as the Federal Aviation Regulations. Common information includes published navigational, airway facility and NOTAM information. Common procedures include published flight procedures such as Instrument Approach Procedures and Standard Terminal Arrival Routes as well as established operational procedures such as airborne holding procedures. The common language of ATM is English with ICAO standard phraseology. The common background and culture has evolved over decades of continuous safety critical interaction. In general it is a culture of professionalism and shared respect between agents. The culture is transmitted through the apprentice training which both Pilots and Controllers experience.

\section{Shared Information in Controller/Pilot Interactions}

The interaction between Pilot and Controller is focused on the management of the specific flight for which they have common responsibility. The principal interaction element is the assigned clearance which constitutes the contractual agreement between the Pilot and Controller for the airspace, runway or airport surface resources assigned to the flight. The need for shared information increases when the clearance must be amended due to some conflict (e.g. weather, traffic, airspace). In an analysis of information required to support shared Controller/Pilot Situation Awareness several key areas have been identified including: weather, traffic, intent and affective states such as workload, urgency or stress. $[4,5]$ Each will be discussed briefly below.

\section{Shared Weather Information}

A primary causal factor for Controller/Pilot interactions is weather, which can make planned trajectories unacceptable for both safety and ride quality reasons. In en route operation, convective weather, turbulence, winds and icing are the major weather factors which must be considered. In terminal and surface operations visibility, ceiling, surface winds and braking action are also key weather factors.

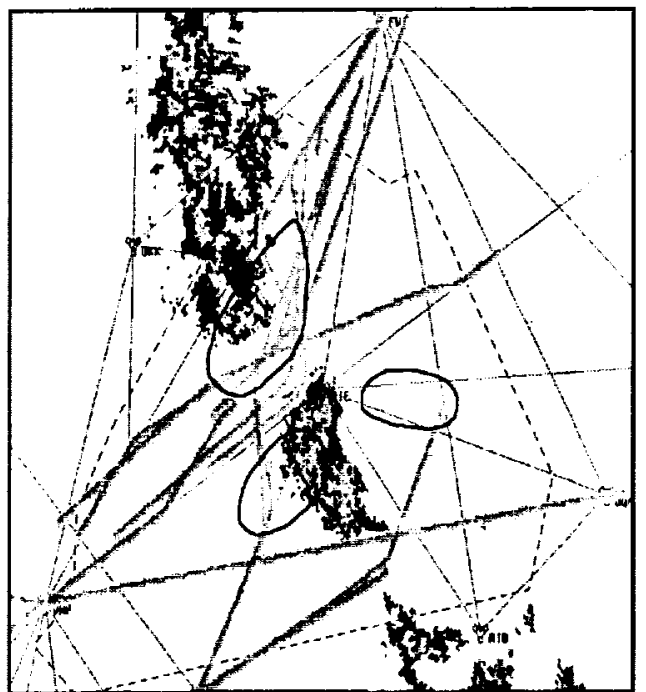

Figure 3: Example of Controller's

inferred weather location (black), aircraft tracks (gray). [14]

In current en route operations, most Pilots have access to airborne weather radar and can visually observe the en route weather whereas the Controllers have limited weather presentation on their displays. As a consequence Controllers rely on Pilot reports of convective weather, turbulence, winds, icing, and deviation requests, to build a mental representation of the spatial extent of weather within their sector. Figure 3 shows examples of an experiment where en route Controllers were asked to draw their mental map of weather impacted areas after controlling traffic around convective weather for approximately 20 min. In most cases the Controllers mental maps captured the major convective areas but there were some gaps and the process is not reliable. [14]

In field observations, Controllers are observed to build similar mental maps for turbulence, icing and winds. Clear Air Turbulence (CAT) is not observable to the Pilots and is a key consideration in route/altitude deviation requests. As a consequence, a collective mental representation of the turbulent regions is built up through informal Pilots' Reports. Access to turbulence information is generally through the Controller's mental representation but is also sometimes obtained through "Party Line" communications. $[8,9]$ The 
principal difficulty in informal PIREP-based weather information is the inconsistency in coverage and age of the information. Often reports are not available, particularly for late night or early morning operations or during the early part of a Controller's shift. Shared representations of wind and icing information are generated by similar processes but are not as common as turbulence reports.

In order to explore the effect of shared weather information on Pilot and Controller interactions, an integrated simulation study was conducted for 6 Pilot/Controller teams in a sector of the Indianapolis Center airspace. In the weather study, active en route Controllers worked high density traffic flows through broken lines of convective weather. In the shared information condition, the Controllers' display included a NEXRAD weather radar overlay which replicated the weather radar information displayed on the Pilot's Electronic Horizontal Situation Indicator (EHSI). The simulated weather scenarios included testable response probes [10] in order to evaluate the Controllers' and Pilots' weather situation awareness. In the case where the Controllers did not have convective weather information displayed, the testable response metric indicated that they were aware of the weather in only $50 \%$ of the cases. In the shared information condition, the results indicate that they were aware in $94 \%$ of the test cases. In $6 \%$ of the cases the results were ambiguous, but there were no cases where the Controllers were clearly unaware of the weather situation. $[4,5]$ In addition to the improved weather situation awareness enabled by the shared information, an improvement in Controller performance was observed in the shared information case. In the 36 test cases, 5 loss of separation events ( 5 miles, $1000 \mathrm{ft}$ ) were observed, all of which occurred in the condition without the shared awareness. The increased performance appears to be due to an improved ability to infer Pilot intent in maneuvering around convective weather ( 2 cases) and a reduction in workload enabled by the enhanced weather situation awareness ( 3 cases). [4,5]

\section{Shared Traffic Information}

Traffic is also a key factor necessitating interactions between Pilots and Controllers. In the analysis of goals and information requirements it was found that Pilots and Controllers have related but differing goals with regard to traffic. The Pilots are observed to have an aircraft-centric view and are primarily concerned with traffic which will impact their current or planned trajectories. Conversely the Controllers have a more system centered, "big picture," view and are concerned with how the trajectories and overall flows will interact. [4,5]

In the current radar controlled environment, Controllers have access to current state information (position, altitude, velocity) of all radar-observed aircraft as well as flight plan information. Unless TCAS-equipped, the Pilot's knowledge of the traffic situation is entirely dependant on being informed by the Controller or from inferred mental representations developed by monitoring the "Party Line". Due to the clear information superiority of the Controller, Pilots will generally defer to Controller's requests with regard to traffic. Controllers will often "point out" proximate traffic both to increase the Pilot's level of traffic situation awareness and to distribute responsibility for traffic separation. If the Pilot acknowledges visually acquiring the rraffic, separation responsibility can be transferred to the Pilot and reduced separation criteria can sometimes be used.

When equipped with TCAS, Pilots have limited information on proximate traffic (typically within $+/$ - $2000 \mathrm{ft}$ altitude and 40 miles) and the potential for shared situation information is increased. As TCAS has become more common and the Pilots and Controllers have gained confidence in the system, the use of TCAS to support shared traffic situation awareness has increased. While there are indications that TCAS provides a basis for shared information, the procedures for TCAS-based interactions have not been fully developed or exploited. Only limited applications of TCASbased interactions, such as passing on oceanic tracks have been approved.

\section{Shared Intent Information}

One of the most useful elements of shared information between Pilots and Controllers is shared information on intent. Past research has advocated the value of intent information in achieving the objective of distributed air-ground traffic management without defining what they consider intent information to be. [11] The current mechanisms for shared intent information are flight plans and published operating procedures. The flight plan is the basic mode of shared information on the goal and intent of the Pilot. The elements of the standard flight plan were developed in the period of (low bandwidth) teletype communication and are quite sparse. The flight plan elements relating to aircraft intent include: 1) Aircraft Identification, 2) Aircraft Type and Equipment, 3) Departure Point, 4) Departure Time, 5) Initial Cruising Altitude, 6) Route of Flight, 7) Airspeed, 8) Destination, 9) Alternate 
Airports, 10) Estimated Time Enroute, and 11) Fuel on Board. $[12,13]$

It is interesting to note that the airway/waypoint structure of the airspace system is designed to efficiently communicate the Route of Flight by both voice and low bandwidth datalink communications. By designating the route clearance in terms of predefined airways, waypoints or procedures (e.g. arrival procedures) both the Pilot and Controller have a shared representation of the clearance. The current airway structure was developed based on the location of ground based navaids and was efficient when air navigation was exclusively based on these navaids. With the proliferation of satellite and inertially based area navigation there is the desire to fly more efficient trajectories. However to be able to communicate the intended trajectory and to coordinate with other Controllers often result in the use of less efficient procedures. There is the need to update the airway system and airspace procedures to take advantage of improvements in navigation and communication capability. However, any future system must be capable of operating in degraded modes and the ability to efficiently articulate the intended routing through voice must be maintained.

At the more tactical level, there is very little direct information on Pilots immediate intentions. The Controller only has the information on the Pilots radar measured states (which exhibit significant lag) and what can be inferred from the flight plan and yoice communications. As a consequence the Controller must allow a significant separation buffer to allow for uncertainty in Pilot intent. In cases when the Pilot's intent is well known (e.g. final approach) it is sometimes possible to reduce the separation criteria. One of the potential advantages of Automatic Dependant Surveillance (ADS) will be a reduction in sampling delay and the possible incorporation of feed forward states indicating aircraft intention such as heading, turn rate or even the progranumed trajectory within the aircraft's Flight Management System.

One study that addressed this issue tested airline Pilots' performance using three traffic displays with varying levels of intent against the basic TCAS display. The first intent display only provided the rate at which the intruder was approaching, shown with an arrow, as well as a conflict probe and a profile view. The second display provided both the commanded altitude and heading of the intruder, and the last display included FMS trajectory including LNAV and VNAV paths. Various performance measures were taken while using each display and it was found that during conflict situations, the Pilots began avoidance maneuvers later using TCAS than both with the Command and Rate displays, and that the Pilots maneuvered later using the Commanded or Rate displays than when using the FMS trajectory display. But the Pilots seemed to find the FMS display the most useful in only the most complicated of situations such as when there was a conflict with two or more aircraft at once. In simpler scenarios, it appeared that the high clutter factor in the FMS display outweighed the benefits of intent information, and the Pilots began their avoidance maneuvers later. So the study seems to have found that a tradeoff between the amount of intent information presented in a display and the clutter it creates has a significant effect on performance. [14]

A final issue in shared intent information is the ability to communicate goals or rationale behind a flight plan request (by the Pilot) or a route change or vector (by the Controller). In current voice communications a Pilot or Controller may provide information supporting the cause of the request. In currently planned CPDLC datalink systems only a limited syntax is expected to be available to provide rationale behind commands or requests.

\section{Shared Affective Information}

An important category of shared information between Pilots and Controllers is Affective information such as emotional state, workload, urgency or capability. [15] In current systems, affective information is inferred from the prosodic content of voice communications. [8] Controllers can clearly communicate urgency or their level of workload by the inflection and rate of their communications. Controllers also routinely assess competency, attentiveness and capability by the speed of response and the prosodic content of Pilot voice transmissions. The importance of shared affective information should be considered in future datalink environments as well as issues regarding the loss of "Party Line" information. Simulator studies of "Party Line" Information usage indicate that PLI is an important but unreliable mode of information transfer. Mechanisms to transmit the important PLI elements should be included in future datalink communication systems such as CPDLC. [8]

\section{Shared Information in Pilot/Airline (PA) Interactions}

During in-flight operations, the Pilot has access to a number of company representatives through voice or datalink (ACARS) communications. In areas of flight planning, the principle interaction 
between the Pilot and the airline is through the Dispatcher who provides flight planning services and supports the Pilots in the event of any disruptions to the planned flight (e.g. weather, delays, diversions). In most airline operations the Dispatcher develops and files the initial flight plan and fuel loading plan which is subsequently reviewed and co-authorized by the Pilot. Pilots frequently amend the fuel load but rarely amend the filed flight plan prior to departure. The flight plan provided to the Pilot is typically a detailed plan which expands on the basic flight plan which is the basis of the Pilot/Controller interaction. The expanded flight plan includes planned altitude changes (step climbs), recommended speeds, as well as weather data. However, in most airlines the rationale behind route planning decisions (made by the Dispatcher) are not communicated to the Pilot directly.

One example of the lack of a shared information basis occurred during the initial phases of the National Route Program (NRP) in the US. The NRP increased the flexibility in high altitude routings for long distance flights and allowed airlines to specify desired routings. Dispatchers typically used the flexibility of the NRP to file "wind optimal" or atherwise optimized routes. Because the rationale was not provided and because the routings used standard waypoints to define the routing, Pilots often requested direct routings which negated the efforts of the Dispatchers and resulted in suboptimal routing. Several airlines issued advisory instructions their Pilots to avoid direct routings when flying NRP flight plans.

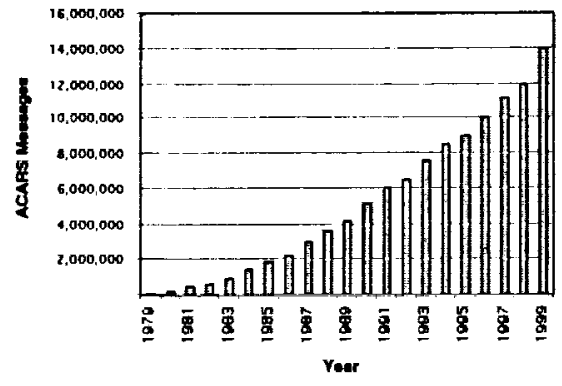

Figure 4: Monthly datalink messages as a function of year. [16]

The increased use of the ACARS datalink can be seen in Figure 4. As datalink has become more popular among airlines, the frequency of information exchange between Pilot and Dispatcher has increased. In the 1980's, the average aircraft sent and received approximately 1,700 messages per month. More recently in the 1990's, that number jumped to 2.700 . [16] When PilotDispatcher interactions were primarily conducted through VHF voice channels, the Pilots and Dispatchers would only communicate if there was a problem. The interaction between Pilot and Controller tended, therefore, to be reactive and during disrupted operations (e.g. hub airport closure) the Dispatcher and communication channel was often overloaded. In the ACARS datalink environment, the interaction between Pilots and Dispatchers appears to have become more pro-active and cooperative. Many Pilots and Dispatchers use ACARS as a low bandwidth email link. It is more common for Pilots to inform dispatch of anticipated problems and for Dispatchers to make suggestions or provide advance information. In large airlines, the Dispatchers and Pilots often sign their ACARS messages creating a personal relationship which was not present before.

The use of ACARS has also influenced Pilot/Airline (PA) interactions between other airline agents such as Maintenance Control or the Local Station. Pilots can communicate with Maintenance for decision support or to alert Maintenance of required maintenance prior to landing. One shared information set between the Pilots and Maintenance for most airlines is a set of maintenance codes which allow efficient transmission of these requests. Local station interactions include gate assignments, "in range" alerting and requests for special services. 7 Shared Information in Intra-Facility
Controller/Controller Interactions

The interaction between Controllers within a single facility tends to focus on coordinating the control of individual flights as well as regulating the flow of aircraft within the facility. Most facilities are arranged so that the primary traffic flow is between adjacent Controllers in order to facilitate interController communication and coordination. This is shown in Figure 5 which schematically represents the flow of aircraft and control responsibility within a tower facility as well as the flow of flight progress strips which are surrogates for the movement of aircraft through the facilities airspace. [17] The flight progress strip contains the flight plan information and is annotated to record changes in flight status other control information. The strips provide a communication and information sharing mechanism between the Radar (R) and Data (D) side Controllers as well as between different sector Controllers and relief 


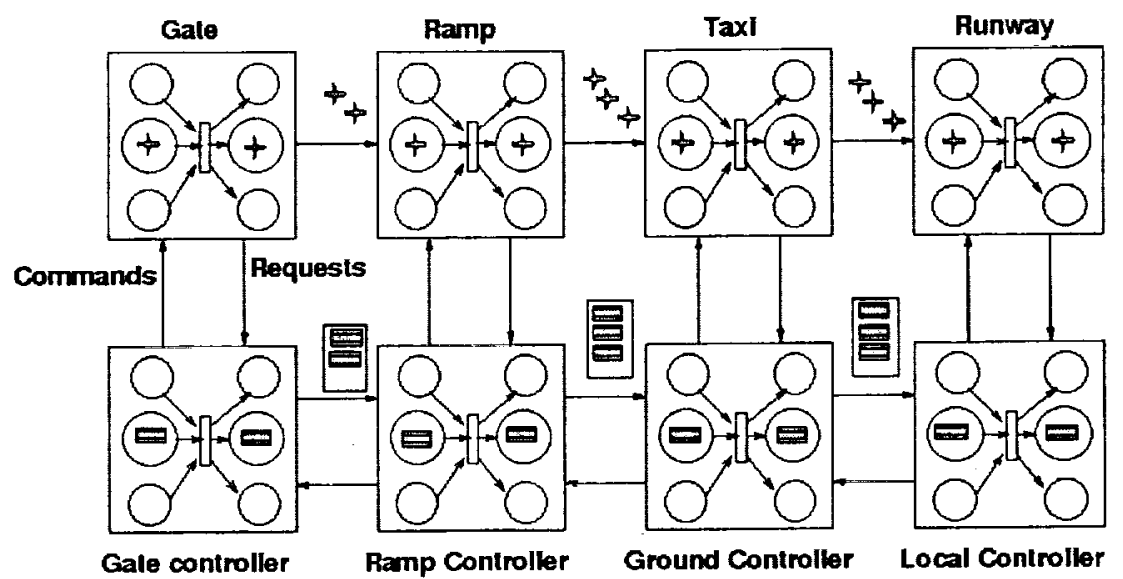

Figure 5: Schematic representation of the flow of aircraft and flight strips through a Tower facility. [17]

Controllers. [18] The transfer of responsibility of aircraft between Controllers is unambiguously represented by physical "handoff" of the flight progress strip. The order of the strips is often a representation of the Controller's planned sequence and intent. In some cases special positioning of the flight progress strip within the strip rack indicate a requirement for special handling. As we discuss increasing the amount of shared information through updating dynamic information in the Host computer, one must also keep in mind the practical concern of the time constraints imposed on the Controller. Even if the information is updated at a reasonable rate, workload induced task-shedding prevents Controllers from reloading the strip information from the Host computer at the same rate. This is an issue with the conflict predictor URET, which integrates flight plan information into its algorithms.

One aspect of Controller/Controller interaction which is often neglected is the affective state of the Controller. Because of their physical proximity, affective state is often communicated by posture, gesture or voice. For example urgency may be transmitted directly by shouting or by a sudden change in posture. Because of safety and working relationship issues, the workload of an adjacent Controller is an important element of shared information. This may be communicated directly or indirectly. For example in Tower operations upstream Controllers may reduce traffic flow based on the accumulation of strips. [17]

In order to improve the interaction between Controllers and underlying automation systems there is a trend to shift from paper flight progress strips to electronic emulation. One advantage of these systems is the potential for inter-Controller communication through the electronic flight progress strip. However, it is important to consider the role of the shared information which is currently communicated in the physical movement of paper strips. In addition, electronic flight progress strips may not be appropriate for some Tower facility control positions which require visual attention to the external scene.

One area in which automation has successfully improved Controller performance is in integrating the tasks of two inter-dependent local Controllers. For example, the Converging Runway Display Aid electronically integrates the aircraft from the two runways on each Controller's runway display through "ghost planes" from the other runway to ease the aircraft converging process. Using this aid, the Controller does not need to acquire the position of the other Controllers' aircraft and mentally integrate the position information with the information with his or her own aircraft. This electronic aid has been successfully integrated into several airports including Boston, Newark, and St. Louis that all use converging runways. [7]

Because most aircraft operations and ATM control actions are routine, an important role of shared information between Controllers is to communicate flight status and intent in irregular cases such as non-standard routings or emergencies. If the Controllers are in close proximity, this is often accomplished by voice communication (sometimes shouting) or gesture. If the Controllers are separated, communication may be through telephone or through an intermediary such as the D-side Controller. 
In some high traffic TRACON facilities, the flow patterns are sufficiently rigid that the simple location of an aircraft along with its radar data block defines its flight plan. In these cases flight progress strips are not used and the shared information is the underlying procedure and flow pattern which provides a shared-control strategy. This system is efficient in minimizing the need for intra-facility communication but makes it very difficult to modify the underlying procedures at the facility level.

An additional important area of shared information is the current and projected state of the facility particularly with regard to traffic and weather. A number of tools have been developed to help supervisors monitor traffic and weather (Weather Radar Displays, ETMS, TMA). These tools have become widely used by Controllers to develop a shared situation awareness at the facility level.

\section{Shared Information in Cross-Facility} Controller/Controller Interactions

The restricted ability of Controllers to interact and share information across facility boundaries limits the flexibility and efficiency of ATM operations. The principle interactions between Controllers, at the single aircraft level, involve coordinating aircraft trajectories, handoffs and special situations. The key mechanisms for information sharing are the Host computer flight information (at the time the flight progress strip is printed), Standard Operating Procedures (SOP) or Letters of Agreement (LOA) between facilities, flow control programs such as Miles In Trail (MIT) restrictions, as well as direct telephone voice communication between sector Controllers.

Because most current cross-facility interaction is bi-lateral it is difficult to coordinate across multiple sectors and there is a restricted ability to accommodate Pilot requests for non-standard routing. Controllers have significant flexibility within their sector but this decreases as the number of involved sectors increases. It is important to consider that the sector issues are threedimensional so the degree of coordination can increase significantly when altitude changes are involved. Even when multi-sector rerouting clearances are given, workload considerations often make it difficult for route amendment to be entered into the host computer. As a consequence it is not uncommon for discrepancies to exist between downstream Controllers' and the Pilots' clearances.

There is a clear need for future systems which support shared information and multi-lateral
Controller interactions to allow more sophisticated use of the airspace in the future. Planned information architectures recognize the need for sharing of flight information across the airspace system. [19] However, there are fundamental issues which must be addressed ranging from how rationale and intent are communicated to how to input workload and how consensus is achieved in multi-lateral electronically-mediated negotiations.

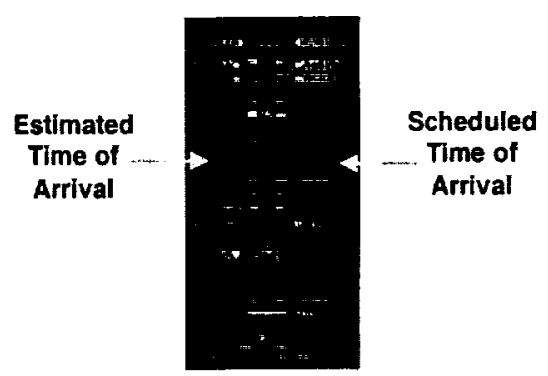

Figure 6: Example of a Traffic Management Advisor (TMA) for use between En Route Center and TRACON. [20]

An additional area of cross-facility interaction is the coordinate operation at the facility level through the Traffic Management Coordinators in each facility. The primary tool for shared information is the Enhanced Traffic Management System (ETMS) which provides data on all airborne traffic and projections for traffic load.

An example of a tool used for information between facilities at the tactical level is the CTAS Traffic Management Advisor (TMA) which is used for the coordination of arrival traffic between Center and TRACON Airspace. An example of the TMA timeline is shown in Figure 6. Both en route and TRACON Controllers have access to the scheduled and estimated times of arrival of aircraft to the feeder fix so that the en route Controllers can provide information to the TRACON about what kind of a flow to anticipate as well as information about cancelled or delayed flights. While NASA designed TMA to perform as a decision aid, optimally sequencing aircraft at the TRACON boundary, a key reported benefit of TMA is improved interaction enabled by the shared information content of TMA. [7] Currently TMA only supports bi-level interaction between a single Center and TRACON. However, development is underway for a Multi-Center TMA which will support multi-lateral interactions. The CTAS technologies have been tested in both Dallas-Ft. Worth and Denver airports. 

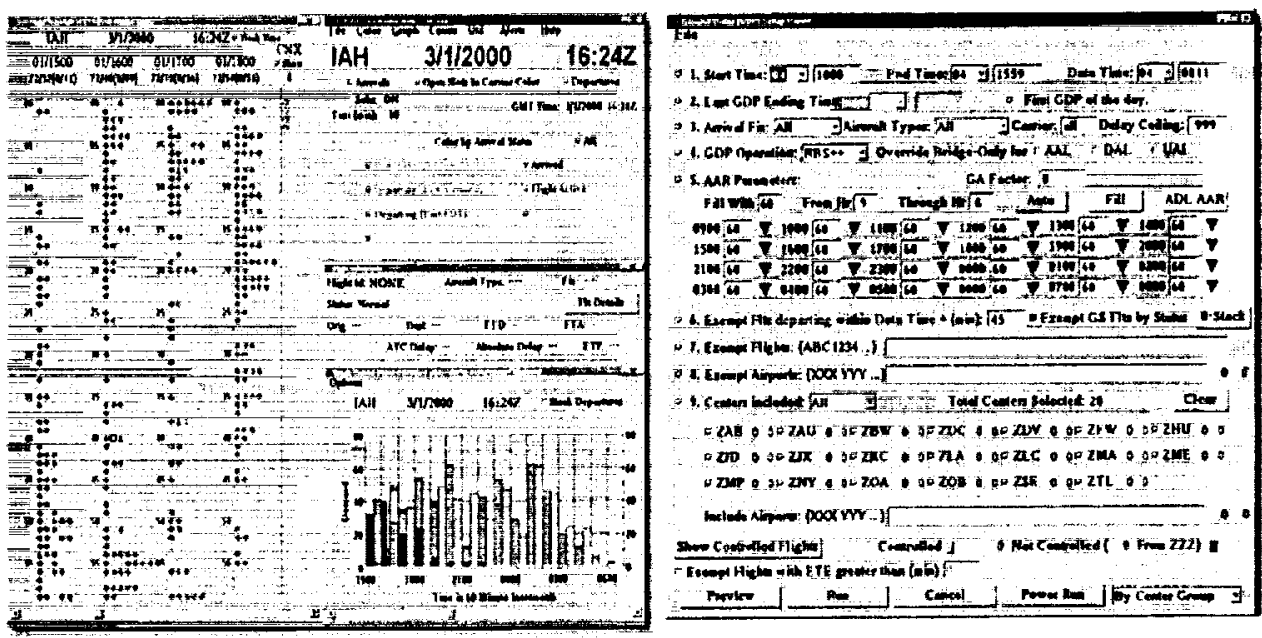

Figure 7: Flight Schedule Monitor predicts traffic flow by using shared information between ATM and AOC. [23]

\section{Shared Information in Airline/ATM Interactions}

The principle basis of shared information between Airlines and ATM are the airline published schedules (e.g. Official Airline Guide) and filed flight plans. Because actual operations often differ from the planned schedule due to weather, equipment or traffic factors, the Controllers often have a poor understanding of the actual plan or intent of the airline. Controllers do not have direct access to the current schedule and gate information available to the public in the terminal, and often must guess if a flight is canceled or delayed if a flight strip is not activated. This is particularly acute during irregular operations.

There are several efforts underway to improve the level of shared information between Airlines and ATM. The most recent effort was an act by the FAA to consolidate weather information between the airlines and the air traffic control facilities through a joint FAAVairline severe weather website. This was a first step in recognizing the importance of shared weather information, however the specific elements of shared weather information have yet to be determined. As further experience in incorporating shared information is gained, the utility of providing specific information will be further established. [21]

At the strategic level, the Collaborative Decision Making (CDM) and the Severe Weather Action Plan (SWAP) provide mechanisms to support information sharing in response to schedule interruptions. The Ground Delay Program also improves overall airline efficiency by allowing the carrier to "swap" Controlled Time of Arrival (CTA) slots with another of that airline's flights. Other strides implementing cooperative problemsolving are the Pacific Track Advisory Program which combines the efforts of the Oakland, California en route center and the airline Dispatchers, and the NRP discussed previously. [22]

One tool used at en route air traffic control facilities is the Flight Schedule Monitor, shown in Figure 7, in which the ATC and the separate AOCs share information about flights throughout the day that have been cancelled or delayed to both predict the traffic flow and become prepared for "heavy flow" periods at a specific airport. The FSM appears to be highly valued by the personnel in the ATC TMU. One TMC comments, "Recent developments and access to new technology has made the job of Traffic Management Coordinator more functional. Implementation of the FSM has provided a real-time data tool pertaining to actual aircraft operations, flight cancellations, and arrival slot re-allocations."

At the tactical level the Surface Movement Advisor (SMA) or the Surface Movement System (SMS) provide shared information on schedule and gate changes as well aircraft in the vicinity of the airport and planned landing runways to coordinate operations on the airport surface. 
The Airport Resource Management Tool (ARMT) is another research effort in which Delta Airlines and Atlanta Hartsfield International Airport (ATL) collaborated to share a computer link to share information about traffic in the terminal environment. Another collaboration, Dynamic Aircraft Route Planning (DARP) recruits the Pilot and air traffic managers in addition to the Controller and Dispatcher to allow aircraft flight plan changes after the aircraft is en route. DARP is intended to be used on trans-Pacific routes to increase fuel efficiency and decrease flight time. [22]

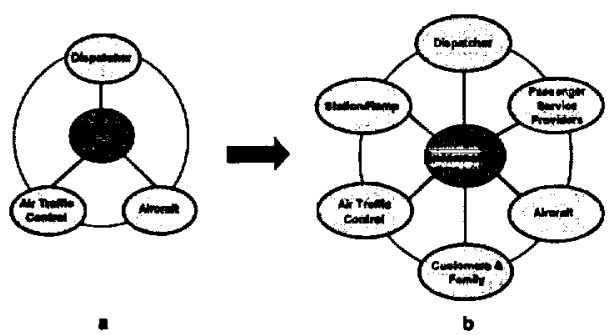

Figure 8: Flight Information Object is a tool for information sharing.

\section{Flight Information Object}

One example of the direction that the future of information sharing is taking is the Flight Information Object (FIO). The FIO is a shared set of information between the Controller, the Pilot and other agents regarding a particular flight, as seen in Figure 8a. The current manifestation of the FIO is the flight plan data in the Host computer. Initial FIO enhancements would be based on data available to the Host computer from planned information tools (e.g., URET, TMA, etc.) and may include additional aircraft data (such as weights, fuel state, etc.). [24]

The FIO concept can be expanded to include access by additional agents who have direct interest in the flight such as the airline station, passenger service providers (e.g., Hotels, Rental Cars, Limos), airline maintenance, etc. The FIO may expand from the Host computer to link additional databases and information sources. At the tactical level, the advanced FIO may include intent and state information linked down from the aircraft autoflight systems by ADS-B or CPDLC. At the airline level, the advanced FIO may include Passenger Itinerary, Cargo Manifest information as well as aircraft maintenance status or other operational information.

\section{Conclusion}

Because of complexity, multiple objectives and the need to be robust to unexpected conditions, ATM will continue to be a distributed multi-agent process with humans executing semistructured decision processes. The key role of advanced information technology in future ATM systems will be to provide mechanisms for information sharing between the human agents which can be expected to improve the collective decision processes within the system.

\section{Acknowledgements}

This work was supported by the NASA Ames Research Center under grants NAG 2-716 and NAG 2-1128 and by TASC as part of the FAA Center of Excellence in Operations Research. The authors acknowledge the contributions of Todd Farley, Mica Endsley, Husni Idris, Ioannis Anagnostakis, Amedeo Odoni, Alan Midkiff, Eric Feron, John-Paul Clarke, Amy Pritchett, and Tom Reynolds.

\section{References}

1. National Airspace System Architecture Version 4.0. DOT, FAA, Washington, DC, January 1999.

2. Kaliardos, William, "Semi-Structured Decision Processes - A Conceptual Framework for Understanding Human-Automation Systems", MTT Ph.D. Thesis, Draper Laboratory Report T-1360, May 1999.

3. Endsley, M., Farley, T., Jones, W., Midkiff, A., and Hansman, R.J., "Situation Awareness Information Requirements for Commercial Airline Pilots," MTT International Center for Air Transportation Report, ICAT-98-1, January 1998.

4. Farley, T., and Hansman, R.J., "An Experimental Study of the Effect of Shared Information on PilovController Re-Route Negotiation," MIT International Center for Air Transportation Report, ICAT-99-1, January 1999.

5. Hansman, R.J., Endsley, M., Farley, T., Vigeant-Langlois, L., and Amonlirdviman, K., "The Effect of Shared Information on Pilot/Controller Situation Awareness and Re-Route Negotiation," EAA/Eurocontrol 2nd International Air Traffic Management R\&D Seminar (ATM 98), Orlando, FL, December 1998.

6. Wickens, Christopher D., Mavor, A.S. and McGee, J.P., ed., Flight to the Future, Human Factors in Air Traffic Control, National Acaderny Press, Washington, DC 1997.

7. Wickens, Christopher D., Mavor, A.S., Parasuraman, R., and McGee, J.P., ed., The Future of Air Traffic Control, Human Operators and 
Automation, National Academy Press, Washington, DC 1998.

8. Midkiff, A., and Hansman, R.J., "Identification of Important 'Party Line' Information Elements and Implications for Situational Awareness in the Datalink Environment," Air Traffic Control Quarterly, 1, No. 1, 5-30, January 1993.

9. Pritchett, A., and Hansman, R.J, "Variations Among Pilots from Different Flight Operations in Party Line Information Requirements for Situation Awareness," Air Traffic Control Quarterly, 4 No. 1, 29-50, January 1997.

10. Pritchett, A., Hansman, R.J., and Johnson, E., "Use of Testable Responses for Performance-Based Measurement of Situation Awareness," International Conference on Experimental Analysis and Measurement of Sitwation Awareness, November 1995.

11. Smith, P.J., Billings, C., Woods, D., McCoy, E., Sarter, N., Denning, R., and Dekker, S., "Can Automation Enable a Cooperative Future ATM System?", Proceedings of the 1997 Aviation Psychology Symposium, 1481-1485, 1997.

12. Air Traffic Control Overview: Kansas Ciry ARTCC, MIT Lincoln Laboratory Group 4I, Air Traffic Automation, FAA, 1998.

13. Air Traffic Controllers Handbook -Air Traffic Control Directive \# 7II0.65L, FAA, Washington, DC, 1998.

14. Barhydt, R. and Hansman, R. J., "Experimental Studies of the Effect of Intent Information on Cockpit Traffic Displays," Journal of Guidance, Control and Dynamics, 22, No. 4, 520-527, Jul./Aug. 1999.

15. Picard, R., Affective Computing, MIT Press, Cambridge, MA, 1997.

16. "GLOBALink/VHF", ARINC Co., presentation prepared for Regional Airline Association Annual Convention, Phoenix, AZ, 1999.

17. Idris, H., Delcaire, B., Hall, W., Anagnostakis, I. Hansman, R.J., Feron, E., and Odoni, A. "Observations of Departure Planning Processes at Logan Airport to Support the Development of Departure Planning Tools," EAAVEurocentrol 2nd International Air Traffic Management R\&D Seminar (ATM 98), Orlando, FL, December 1998. 18. Mackay, W., Fayard, A., Frobertam, L., Medini, L., "Reinventing the Familiar: Exploring an Augmented Reality Design Space for Air Traffic Control, Proceedings of the ACM CHI' $98,1998$.
19. "A Concept of Operations for the National Airspace System in 2005", revision 1.3, FAA, June 27, 1997.

20. NASA CTAS website, "Traffic Management Advisor", http://ctas.arc.nasa.gov.

21. FAA Office of Public Affairs, "President Unveils Severe Weather Plan, DOT Cites Unprecedented FAA and Industry Collaboration and Launches New Air Traffic Information Website,"http://www.faa.gov/apa/pr/pr.cfm?id=99 3, March, 2000.

22. Airline Operational Control Overview, prepared by Airline Dispatchers Federation and Seagull Technology, Inc., Cooperative Research and Development Agreement 93-CRDA-0034, September, 1995.

23. Metron, Inc. website, "Flight Schedule Monitor", http://www.metsci.com/cdm/fsm.html.

24. Taber, N. J. \& Viets, K., "Flight Object Operational Concept" (draft version), The MTTRE Corporation, McLean, VA, March 2000.

\section{Biographical Note}

R. John Hansman has been on the faculty of the Department of Aeronautics \& Astronautics at MIT since 1982. He obtained his A.B. in Physics from Cornell University in 1976, his S.M. in Physics in 1980 and his Ph.D. in Physics, Meteorology, Aeronautics \& Astronautics and Electrical Engineering from MIT in 1982. He is the Head of the Humans and Automation Division, the Director of the MIT International Center for Air Transportation and of the Aeronautics Systems Laboratory. He conducts research in several areas related to flight vehicle operations and aviation safety. His current research activities focus on advanced cockpit information systems, including Flight Management Systems, air-ground datalink, electronic charting, advanced alerting systems, and flight crew situational awareness, cockpit human factors and information management.

Hayley J. Davison is a Masters student in the International Center for Air Transportation of the Department of Aeronautics \& Astronautics at MIT. She obtained her B. S. in Psychology from the University of Illinois in 1999. Her main research interests include aviation human factors, advanced information systems, automation issues, and flight deck displays. 